\title{
Análise das fontes de informação sobre os autoexames da mama disponíveis na Internet
}

\author{
Analysis of information sources about breast self examination \\ available on the internet
}

Marcia Regina Cubas ${ }^{1}$

Paulo Cesar Zimmermann Felchner ${ }^{1}$

${ }^{1}$ Escola Politécnica, Pontifícia Universidade Católica do Paraná. Rua Imaculada Conceição 1155, Prado Velho. 80215-901 Curitiba PR. m.cubas@pucpr.br

\begin{abstract}
Objective: To analyze the information about breast self examination available on the Internet. Methods: A descriptive documental study was performed via a search on the Google and Yahoo websites using the phrase "breast self examination" in Portuguese, and the first 50 results from each site were analyzed using the criteria of the American Medical Association and Health on the Net. Results: 68 sites were selected and analyzed. Most of the sites were in the commercial domain, six were governmental sites and five were Portuguese sites. More than half had restrictions regarding criteria of authority and authorship; $61 \%$ did not have contact details and $52.94 \%$ considered breast self exam as part of a set of measures; $26.47 \%$ had correct and complete evidence-based content; $33.82 \%$ had context references. Government WebPages of health or professional institutions had restrictions regarding content or presentation. Conclusions: The information about breast self exam is heterogeneous. Only a small number of websites were concerned about quality criteria, both in terms of construction and content. The bulk of the information available is not evidence-based and there is potentially dangerous information for the patient. It is necessary to improve the quality of websites dealing with breast self exam.
\end{abstract}

Key words Breast self exam, Internet, Consumer health information
Resumo Objetivo: Avaliar as informações sobre autoexame da mama disponíveis ao público da internet. Método: Estudo descritivo documental, cujo universo foi composto pelos sites "Google" $e$ "Yaho"", utilizando-se o descritor "autoexame de mama". Buscaram-se os 50 primeiros resultados. As páginas Web foram analisadas pelos critérios da American Medical Association e da Health on the Net. Resultados: Foram selecionadas e analisadas 68 páginas. A maioria dos sites era de domínio comercial, seis governamentais e cinco portugueses. Mais da metade tinham restrições quanto ao critério de autoridade e autoria; $61 \%$ não apresentavam meio de contato e $52,94 \%$ consideravam o autoexame como parte de um conjunto de medidas; $26,47 \%$ possuíam o conteúdo baseado em evidências, preciso e completo; $33,82 \%$ possuíam referências dos conteúdos. Páginas-Web governamentais, de instituições de saúde ou profissionais possuíam restriçães com relação ao conteúdo ou apresentação. Conclusões: As informações sobre autoexame de mama são heterogêneas. Poucas páginas demonstraram preocupação com critérios de qualidade, tanto da construção quanto do conteúdo. A maior parte das informações não está baseada em evidências e que podem trazer danos ao paciente. Há necessidade de melhorar a qualidade das páginas Web que abordam o tema.

Palavras-chave Autoexame de mama, Internet, Informação de saúde ao consumidor 


\section{Introdução}

O câncer de mama é um problema de saúde pública. Trata-se do segundo tipo de câncer mais frequente em todo o mundo e o mais incidente no sexo feminino, sendo responsável por cerca de $22 \%$ dos casos novos de câncer em mulheres. Podendo apresentar um prognóstico relativamente bom quando diagnosticado em estádios iniciais ${ }^{1,2}$.

É a neoplasia mais temida devido aos seus efeitos psicológicos de alteração da autoimagem e da sexualidade ${ }^{3,4}$. Possui elevada mortalidade e é a doença que mais demanda internações em mulheres, além do alto custo de seu tratamento ${ }^{5}$.

$\mathrm{Na}$ América, Latina a incidência do câncer de mama, assim como a sua mortalidade, vem aumentando ${ }^{6}$, enquanto que em países desenvolvidos a mortalidade mostra uma tendência para a diminuição ${ }^{7}$. No Brasil, estima-se que ocorreram, em 2010, cerca de 49.240 casos novos de câncer de mama, com risco de 49 casos para cada 100.000 mulheres $^{2}$.

O status socioeconômico elevado é uma característica associada a essa doença. Entre os fatores de risco comuns, principalmente nos Estados Unidos, Europa e América Latina, destacamse: a idade avançada; os fatores ambientais, hormonais, reprodutivos e genéticos; o sedentarismo; a ingestão de gordura; e a obesidade ${ }^{8,9}$.

Apesar do conhecimento dos fatores associados à neoplasia, cerca de $60 \%$ dos casos, no Brasil, são diagnosticados em estádios avançados 9 . Sabe-se que a mamografia é o instrumento mais efetivo para o diagnóstico precoce, mas é pequeno o número de mulheres que realizam esse exame no país e menor número, ainda, o de idosas que o realizam. Alguns fatores podem influenciar a sua realização, como educação, nível socioeconômico, renda familiar e local de domicílio ${ }^{10}$.

O prognóstico do câncer de mama será influenciado por diversos fatores que podem alterar a seleção do tratamento. Dentre eles estão a idade e o estadiamento da doença ${ }^{11}$. Quanto mais precoce for feito o diagnóstico, maior a chance de cura. Assim, o objetivo principal é a detecção de doença assintomática, que possui um prognóstico mais favorável, além de necessitar de tratamentos menos agressivos ${ }^{12}$. Desde o início do desenvolvimento da neoplasia até o momento em que ela é detectável no exame clínico, com cerca de um (1) cm, passam-se 10 anos, em média, havendo duplicação do seu tamanho a cada três ou quatro meses ${ }^{13}$.

O autoexame da mama pode ser uma opção para a detecção da neoplasia mamária ${ }^{4}$, sendo recomendado pelo consenso do Ministério da Saúde/Instituto Nacional do Câncer (INCA), porém não tem demonstrado diminuição da mortalidade por câncer de mama nas pacientes que o realizam ${ }^{14}$. Em 2007, o INCA passa a esclarecer a restrição sobre o uso do autoexame das mamas. Alerta que este deve estar associado às demais formas de detecção precoce e ressalta possíveis efeitos negativos do autoexame, como a realização de biópsias desnecessárias. Assim, seu papel é controverso na detecção precoce da neoplasia mamária, sendo recomendado o esclarecimento à paciente sobre os potenciais benefícios e também suas limitações ${ }^{15}$. Em um estudo brasileiro, que verificou o conhecimento sobre o assunto, evidenciou-se que somente 7,4\% das mulheres tinham conhecimento adequado sobre o autoexame da mama ${ }^{16}$, corroborando com a necessidade de atenção a este fato.

A internet é fonte de informação cada vez mais utilizada globalmente, sendo que muitas mulheres procuram informações sobre saúde na rede, utilizando ferramentas de busca de uso geral, de maneira aleatória e sem ajuda de profissionais de saúde. Estima-se que haja mais de 100.000 web sites em saúde na internet, que variam desde altamente acadêmicos, periódicos com arbitragem, sites governamentais e de instituições de saúde até sites de contribuição individual de pacientes ou profissionais ${ }^{17}$.

As informações, oferta de serviços e produtos na internet tem o potencial de promover a saúde, mas também pode causar danos ao usuário, como tratamentos incorretos ou adiamento de cuidados em saúde ${ }^{17,18}$. A qualidade e a confiabilidade de tais informações são fundamentais para que a mulher possa ter um cuidado adequado à sua saúde. Em situações de repasse inadequado das informações pode haver prejuízo para sua saúde, sendo mais chocante nos casos de doenças neoplásicas que são acompanhadas de estigmas.

Neste contexto, o objetivo do presente artigo é avaliar as informações sobre autoexame da mama disponíveis ao público da internet.

\section{Método}

Trata-se de um estudo descritivo documental que teve como universo de pesquisa os sites de busca: "Google" e "Yahoo". Foi utilizado na busca o Descritor em Ciência da Saúde: "autoexame de mama" e selecionados os 50 primeiros resultados da pesquisa de cada site. A busca foi realiza- 
da em Dezembro de 2008 e em Janeiro de 2009. Incluíram-se para a análise todas as páginas que abordavam o autoexame de mama. Sites que estavam duplicados foram considerados uma única vez. Os dados coletados foram tabulados em planilha eletrônica do Microsoft Excel ${ }^{\circledR}$.

A análise foi baseada nos critérios da American Medical Association (AMA) e da Health on the Net (HON), obtidos por documento da Sociedade Brasileira de Informática em Saúde (SBIS) ${ }^{17}$, cujos itens abrangem: a) a autoridade: deve incluir profissionais qualificados ou a referência de que a informação foi fornecida por pessoa não qualificada na área da saúde; b) a complementariedade: informação deve ser complementar e não substitui o aconselhamento médico; c) as informações devem ser baseadas em evidências; d) o conteúdo deve ser preciso e completo; e) deve informar a autoria do conteúdo; f) deve conter as datas de publicação inicial e de atualização.

Partindo dos critérios citados, o conteúdo das páginas foi classificado da seguinte forma: a) autoridade: presença ou ausência; b) complementariedade: sim, não ou parcial (quando a informação contida na página é contraditória ou deixa dúvidas); c) baseadas em evidências: sim, não ou parcial (quando parte das informações não são ancoradas cientificamente); d) conteúdo: preciso e completo, impreciso e incompleto, preciso e incompleto, impreciso e completo (sendo estes dois últimos considerados como parcial); e) autoria: presença ou ausência; f) datas: presença ou ausência.

Os resultados foram apresentados quantitativamente, com frequência absoluta e relativa. Como padrão de comparação do conteúdo utilizou-se a recomendação para detecção precoce elaborada pelo $\mathrm{INCA}^{13}$.

\section{Resultados}

Das 100 páginas selecionadas, 15 se repetiam nos dois sites; uma apareceu duas vezes num mesmo site; sete estavam relacionadas diretamente ao meio acadêmico, trazendo resumos ou artigos completos de difícil assimilação e interpretação para o público; e 10 não traziam informações sobre o autoexame de mama, apenas citavam-no, faziam propaganda de produtos, eram páginas suspensas ou falavam sobre o exame clínico da mama. No entanto, uma delas ressaltava que o INCA não recomenda o autoexame como medida isolada.

Com a aplicação do critério acima descrito, foram utilizados 68 sites para análise. Dentre eles, a grande maioria era de domínio comercial $(n=49)$; os seis governamentais se dividiam em páginas de governo estrangeiro, brasileiro (união, estado e município) e INCA; cinco eram portugueses; e os outros se distribuíam em universidades, organizações e páginas de profissionais de saúde (Figura 1).

Em relação aos critérios de autoridade e autoria, 35 sites $(51,47 \%)$ não indicavam autor qualificado para fornecer a informação ou omitiam a não qualificação do responsável. Considerouse que as empresas de saúde seriam qualificadas para o fornecimento das informações.

A maior parte dos sites $(61,76 \%)$, não apresentava um meio de contato para ser usado pelo internauta, como endereço eletrônico ou telefone.

Quanto ao critério de complementaridade da informação ao aconselhamento médico, 36 $(52,94 \%)$ indicavam ser o autoexame da mama parte de um conjunto de medidas; 29 sites (42,65\%) não apresentavam esta informação de maneira clara; e três $(4,41 \%)$ faziam-na parcialmente.

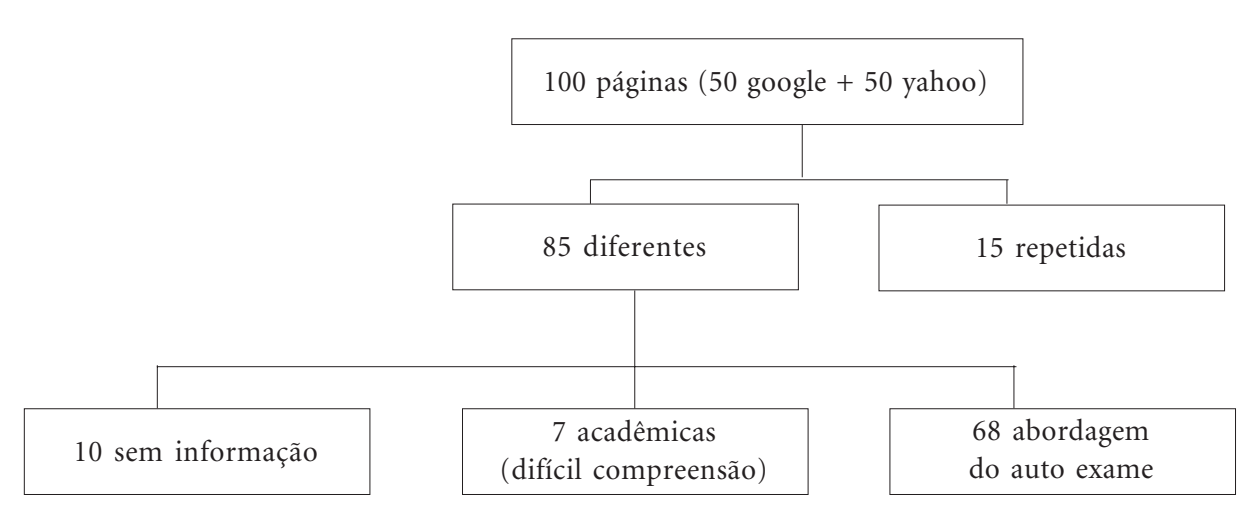

Figura 1. Seleção das páginas WEB para análise das fontes de informação sobre autoexame de mama, 2009. 
O conteúdo baseado em evidências e as limitações do autoexame da mama e seus benefícios não foram contemplados em 46 páginas $(67,65 \%)$; quatro $(5,88 \%)$ mostravam tais informações de maneira parcial; e 18 (26,47\%) mostravam conteúdos baseados em evidências e apresentavam as limitações do autoexame da mama.

Quanto aos critérios de precisão e completude, 28 páginas Web $(41,18 \%)$ não atendiam a estes critérios; $22(32,35 \%)$ o faziam de maneira parcial; e $18(26,47 \%)$ tinham o conteúdo preciso e completo.

Dentre os domínios governamentais (.gov), o site do INCA possuía informações adequadas e completas, enquanto que quatro sites informavam parcialmente e uma página não possuía informações adequadas. Em meio às páginas de universidades, duas apresentavam conteúdo adequado e completo, e uma não atendia a nenhum dos critérios. Este mesmo perfil foi verificado entre as organizações sem fins lucrativos.

No que se refere às páginas de domínio de Portugal (.pt), três eram relacionados a entidades de saúde, no entanto apenas uma delas apresentava conteúdo adequado e completo.

$\mathrm{O}$ site de propriedade de um profissional médico não possuía informações adequadas.

Vinte e três páginas $(33,82 \%)$ possuíam referências de seus conteúdos e a indicação da data de publicação e de atualização dos conteúdos estava presente em seis páginas $(8,82 \%)$, no entanto, 27 sites $(39,71 \%)$ possuíam ao menos uma das duas informações.

\section{Discussão}

O uso de tecnologias de informações pode ser importante para a educação de profissionais e pacientes em várias áreas da saúde ${ }^{19,20}$ e o uso da internet para aquisição de informações por parte dos pacientes tem aumentado gradualmente ${ }^{21}$. A busca na internet sobre o autoexame da mama tem potencialidade para trazer informações incorretas ou incompletas, induzindo o internauta, o que pode resultar em prejuízo à saúde ou promover um diagnóstico tardio do câncer de mama.

A informação da autoridade das páginas analisadas está de acordo com outros estudos, nos quais foram identificadas em $27 \%$ das fontes as credenciais dos autores e em $61 \%$ o nome da instituição responsável pela mesma ${ }^{22}$. Este fato remete à discussão de que páginas podem ser consideradas suspeitas quando não divulgam a formação técnica do autor e a instituição a qual pertence, pois não oferece reconhecimento, bem como não se responsabiliza pela informação.

Se por um lado, a busca de informações fornece às pessoas maior possibilidade de acesso ao conhecimento, de metabolizá-lo e de incorporálo a mudanças nas suas práticas de saúde e, portanto, de não aceitar passivamente determinações médicas, consequentemente, de sentirem-se capazes de decidir sobre seu diagnóstico e tratamento, por outro lado, ao acessar informações contraditórias, incorretas ou fraudulentas ${ }^{23}$, o benéfico processo de mudança pode estar comprometido e resultar em prejuízo à saúde do indivíduo. Além disso, algumas fontes de informação são veículos de empresas que desejam somente promover algum produto ${ }^{23}$.

O autoexame das mamas é muito divulgado para o público em geral e encontra-se em grande parte das publicações da internet. Mesmo sites com informações dadas por profissionais habilitados trazem informações incompletas ou incorretas, o que foi verificado neste estudo. Este fato foi também encontrado em estudo de metodologia semelhante que avaliou informações sobre obesidade infantil ${ }^{7}$.

O autoexame não é recomendado para uso isolado. Deve-se enfatizar que ele faz parte de um conjunto de medidas. Estudo que abordou a qualidade de informações sobre câncer de mama na internet refere que $25 \%$ das páginas apenas avisavam que as informações não substituem a consulta médica ${ }^{24}$. Neste contexto, os achados do nosso estudo de que $52,94 \%$ das páginas Web explicitam a complementaridade do autoexame, reforça a ideia, e a consequente atitude, de que o autoexame de mama, usado isoladamente, é capaz de detectar o agravo. De maneira mais grave, havia a indicação para a procura do médico somente se a paciente tivesse alteração no autoexame, informação esta que pode ser danosa, pois ao palpar a lesão maligna, a neoplasia já se encontrará com prognóstico mais sombrio, sendo necessário que mulheres assintomáticas façam avaliação médica periódica.

O percentual de $26 \%$ de páginas que apresentava informações baseadas em evidências científicas foi semelhante ao encontrado em outro estudo nacional que avaliava a qualidade das informações sobre rinite alérgica ${ }^{25}$.

Dentre os 55 sites comerciais, 11 apresentaram conteúdo adequado e completo, 16 parcial e 23 inadequado e incompleto. Esse achado concorda com outro estudo que avaliou informações sobre leishmaniose visceral na internet e verificou grande quantidade de informações ausentes ${ }^{26}$. 
Muitos portais governamentais direcionados à saúde trazem informações com o intuito de auxiliar os indivíduos a participarem da decisão sobre sua saúde e a influenciar tanto na prevenção como no diagnóstico e no tratamento. Além disso, os governos têm, a princípio, profissionais com capacidade técnica adequada, que não forneceriam informações inadequadas. Porém, o que se verificou neste estudo é que dos seis sites governamentais somente o INCA trazia informações completas e corretas. Quatro deles tinham conteúdo parcialmente completo e correto e um trazia informações incorretas. Este fato não é exclusividade brasileira, pois estudo que avaliou quatro portais governamentais de língua inglesa encontrou neles informações não claras, incompletas ou confusas ${ }^{27}$.

Nas páginas não comerciais verificou-se qualidade de informação heterogênea. Metade possuía informações adequadas e completas, sendo que uma página oficial do governo apresentava informações incorretas. Cabe discutir que dois dos sites governamentais são de funções jurídicas, portanto, com atuação principal fora da área da saúde, o que pode explicar, em parte, a inadequação do conhecimento. Tais dados contrastam com estudo sobre leishmaniose visceral que encontrou melhor qualidade de informações em sites governamentais ${ }^{26}$.

Com relação às orientações, um site informava que o autoexame previne o câncer de mama e nove referiam que o mesmo faz diagnóstico precoce ou é o melhor método para o diagnóstico inicial. Somente quatro destacaram possíveis efeitos negativos do autoexame. Este conjunto de dados disponíveis na internet pode gerar informações sobre saúde incompletas, não claras e podem trazer confusão ao paciente ${ }^{18}$.

A data de publicação e a atualização foi referida em seis $(8,82 \%)$ páginas Web e trinta $(44,12 \%)$ apresentavam ou a data de publicação ou a da atualização das informações. Neste ponto, outros estudos oferecem heterogeneidade que vai desde os $45 \%{ }^{25}$ a $13 \%$ das páginas com data de elaboração ${ }^{22}$ e com percentual pouco inferior em avaliação de informações em saúde na internet em sites aparentemente confiáveis ${ }^{28}$.

\section{Conclusão}

A internet é um campo de fácil e crescente acesso a informações, consequentemente, as relativas à saúde estão ao alcance de todos os indivíduos, com potencial de auxiliar na decisão sobre condutas para prevenção, diagnóstico e tratamento.

No entanto, ao analisar as informações sobre autoexame de mama, observa-se que poucas páginas Web demonstraram preocupação com critérios de qualidade, tanto da construção do site quanto das informações fornecidas, as quais, na sua maioria não estavam baseadas em evidências e algumas delas com risco de danos à saúde de quem as acessa. Considera-se premente a necessidade de melhorar a qualidade das páginas Web que informam sobre o autoexame de mama, principalmente, as de responsabilidade governamental.

\section{Colaboradores}

MR Cubas e PCZ Felchner participaram igualmente de todas as etapas de elaboração do artigo.

\section{Referências}

1. Brasil. Ministério da Saúde (MS). Secretaria de Atenção à Saúde. Instituto Nacional de Câncer (INCA). Coordenação de Prevenção e Vigilância de Câncer. Estimativas 2008: incidência de câncer no Brasil. Rio de Janeiro: Instituto Nacional de Câncer (INCA); 2007. [acessado 2012 mar 15]. Disponível em: http:/ /bvsms.saude.gov.br/bvs/publicacoes/estimativa incidencia_cancer_2008.pdf

2. Brasil. Ministério da Saúde (MS). Instituto Nacional de Câncer (INCA). Estimativas 2010: incidência de câncer no Brasil. Rio de Janeiro: Instituto Nacional de Câncer (INCA); 2009. 
3. Brasil. Ministério da Saúde (MS). Secretaria de Atenção à Saúde. Instituto Nacional de Câncer (INCA). Coordenação de Prevenção e Vigilância. A situação do câncer no Brasil. Rio de Janeiro: Instituto Nacional de Câncer (INCA); 2006. [acessado 2012 mar 15]. Disponível em: http://iah.iec.pa.gov.br/iah/ fulltext/pc/monografias/ms/situcancerbrasil/ situcancerbras2006.pdf

4. Brasil. Ministério da Saúde (MS). Secretaria Nacional de Assistência à Saúde. Instituto Nacional de Câncer (INCA). Coordenação de Prevenção e Vigilância. Controle do câncer de mama: documento de consenso. Rio de Janeiro: Instituto Nacional de Câncer (INCA); 2004. [acessado 2012 mar 15]. Disponível em: http://www.inca.gov.br/publicacoes/ Consensointegra.pdf

5. Boing AF, Vargas SAL, Boing AC. A carga das neoplasias no Brasil: mortalidade e morbidade hospitalar entre 2002-2004. Rev Assoc Med Bras 2010; 53(4):317-322.

6. Guerra MR, Gallo CVM, Mendonça GAS. Risco de câncer no Brasil: tendências e estudos epidemiológicos mais recentes. Rev Bras Cancerol 2005; 51(3):227-234

7. Paulinelli RR, Freitas Jr R, Curado MP, Souza AA. A situação do câncer de mama em Goiás, No Brasil e no mundo: tendências atuais para a incidência e a mortalidade. Rev Bras Saude Mater Infant 2003; $3(1): 17-24$.

8. Cuevas SAR, Garcia MC. Epidemiología del cancér de mama. Ginecol Obstet Mex 2006; 74(11):585-593.

9. Pinho VFS, Coutinho, ESF. Variáveis associadas ao câncer de mama em usuárias de unidades básicas de saúde. Cad Saude Publica 2007; 23:1061-1069.

10. Veras R. Envelhecimento populacional e as informações de saúde do PNAD: demandas e desafios contemporâneos. Introdução. Cad Saude Publica 2007; 23(10):2463-2466.

11. National Cancer Institute. United States National Institutes of Health. Breast cancer treatment $\left(P D Q^{\circledR}\right)$. [acessado 2012 mar 15]. Disponível em: http:// www.cancer.gov/cancertopics/pdq/treatment/breast/healthprofessional/allpages

12. Armstrong K, Moye E, Williams S, Berlin JA, Reynolds EE. Screening mammography in women 40 to 49 years of age: a systematic review for the American College of Physicians. Ann Intern Med 2007; 146(7):516-526.

13. Brasil. Ministério da Saúde (MS). Instituto Nacional do Câncer (INCA). Deteç̧ão precoce do câncer de mama. [acessado 2012 mar 15]. Disponível em: http://www.inca.gov.br/conteudo_view.asp?id=1932

14. Smith RA, Cokkinides V, Browley OW. Cancer screening in the United States, 2008: a review of current american cancer society guidelines and cancer screening issues. CA Cancer J Clin 2008; 58(3):161-79.

15. Rim A, Chellman-Jeffers M. Trends in breast cancer screening and diagnosis. Cleve Clin J Med. 2008; 75(Supl.1):S2-9.

16. Marinho LAB, Costa-Gurgel MS, Cecatti JG, Osis MJD. Conhecimento, atitude e prática do autoexame das mamas em centros de saúde. Rev Saude Publica 2003; 37(5):576-582.
17. Juzzo LMLC. Critérios para avaliação da qualidade das informações sobre saúde disponíveis online. In: Anais do IX CBIS; 2004; Ribeirão Preto. [acessado 2012 mar 15]. Disponível em: http://www.imeo. com.br/artigos/inform-saude.pdf

18. Glenton C, Paulsen EJ, Oxman AD. Portals to wonderland: health portals lead to confusing information about the effects of health car. BMC Medical Informatics and Decision Making. 2005 Mar [acessado 2012 mar 15];5(7). Disponível em: http:// www.biomedcentral.com/1472-6947/5/7

19. Marques IR, Marin HF. Enfermagem na Web: processo de criação e validação de um Web site sobre doença arterial coronariana. Rev Latino-am Enfermagem 2002;10(3):298-307.

20. Santos SGF, Marques IR. Uso dos recursos de internet na enfermagem: uma revisão. Rev Bras Enferm. 2006; 59(2):212-216.

21. Kummervold PE, Chronaki CE, Lausen B, Prokosch HU, Rasmussen J, Santana S, Staniszewski a, Wangberg SC. eHealth Trends in Europe 2005-2007: A Population-Based Survey. J Med Internet Res. 2008; 10(4):e42.

22. Silva EV, Castro LLC, Cymrot R. Tratamento farmacológico da obesidade em páginas da internet brasileira: análise dos critérios técnicos de qualidade. Rev Ciênc Farm Básica Apl. 2008; 59(2):159-165.

23. Garbin HBR, Pereira Neto AFP, Guilam MCR. A internet, o paciente expert e a prática médica: uma análise bibliográfica. Interface Comunic Saúde Educ 2008; 12(26):579-588.

24. Meric F, Bernstam EV, Mirza NQ, Hunt KK, Ames FC, Ross MI, Kuerer HM, Pollock RE, Musen MA, Singletary E. Breast cancer on the world wide web: cross sectional surveyof quality of information and popularity of websites. BMJ 2002; 324(7337):577-581.

25. Silva LVER, Mello Júnior JF, Mion O. Avaliação das informações sobre rinite alérgica em sites brasileiros na rede mundial de computadores (Internet). Rev Bras Otorrinolaringol. 2005; 71(5):590-597.

26. Souza CLN, Luz ZP, Rabello A. Análise da informação sobre a leishmaniose visceral disponível em portais brasileiros da rede mundial de computadores - Internet. Revista da Sociedade Brasileira de Medicina Tropical. 2008; 41(4):352-357.

27. Demiris G, Afrin LB, Speedie S, Courtney KL, Sondhi M, Vimarlund V, Lovis C, Goossen W, Lynch C. Patient-centered applications: use of information technology to promote disease management and wellness. a white paper by the amia knowledge in motion working group. J Am Med Inform Assoc. 2008; 15(1):8-13.

28. Kunst H, Groot D, Latthe PM, Latthe M, Khan KS. Accuracy of information on apparently credible websites: survey of five common health topics. $B M J$ 2002; 324(7337):581-582.

Artigo apresentado em 13/09/2010

Aprovado em 20/12/2010

Versão final apresentada em 10/01/2011 